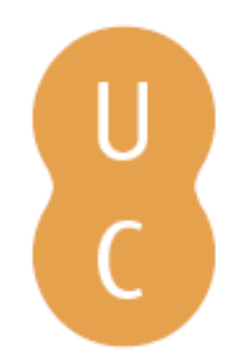

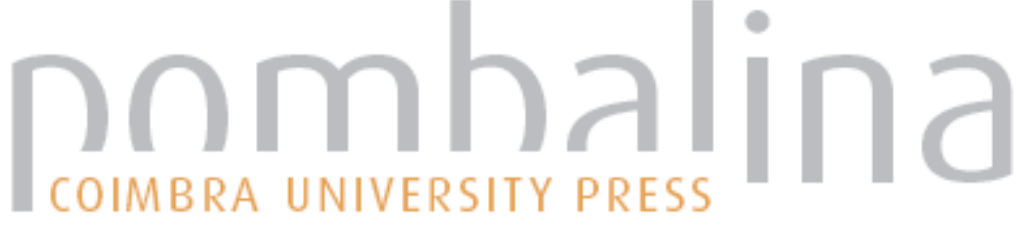

\section{La divulgación científica en el entorno universitario}

Autor(es): $\quad$ Lascurain Sánchez, María Luisa; Sanz Casado, Elías

Publicado por: Imprensa da Universidade de Coimbra

URL

persistente:

URI:http://hdl.handle.net/10316.2/31964

DOI:

DOI:http://dx.doi.org/10.14195/978-989-26-0319-3_40

Accessed : $\quad$ 26-Apr-2023 08:59:35

A navegação consulta e descarregamento dos títulos inseridos nas Bibliotecas Digitais UC Digitalis, UC Pombalina e UC Impactum, pressupõem a aceitação plena e sem reservas dos Termos e Condições de Uso destas Bibliotecas Digitais, disponíveis em https://digitalis.uc.pt/pt-pt/termos.

Conforme exposto nos referidos Termos e Condições de Uso, o descarregamento de títulos de acesso restrito requer uma licença válida de autorização devendo o utilizador aceder ao(s) documento(s) a partir de um endereço de IP da instituição detentora da supramencionada licença.

Ao utilizador é apenas permitido o descarregamento para uso pessoal, pelo que o emprego do(s) título(s) descarregado(s) para outro fim, designadamente comercial, carece de autorização do respetivo autor ou editor da obra.

Na medida em que todas as obras da UC Digitalis se encontram protegidas pelo Código do Direito de Autor e Direitos Conexos e demais legislação aplicável, toda a cópia, parcial ou total, deste documento, nos casos em que é legalmente admitida, deverá conter ou fazer-se acompanhar por este aviso.

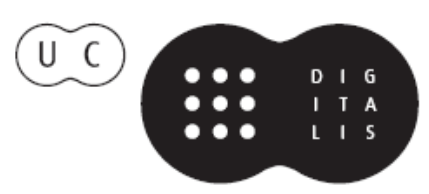


Maria Manuel Borges

Elias Sanz Casado

Coordenação

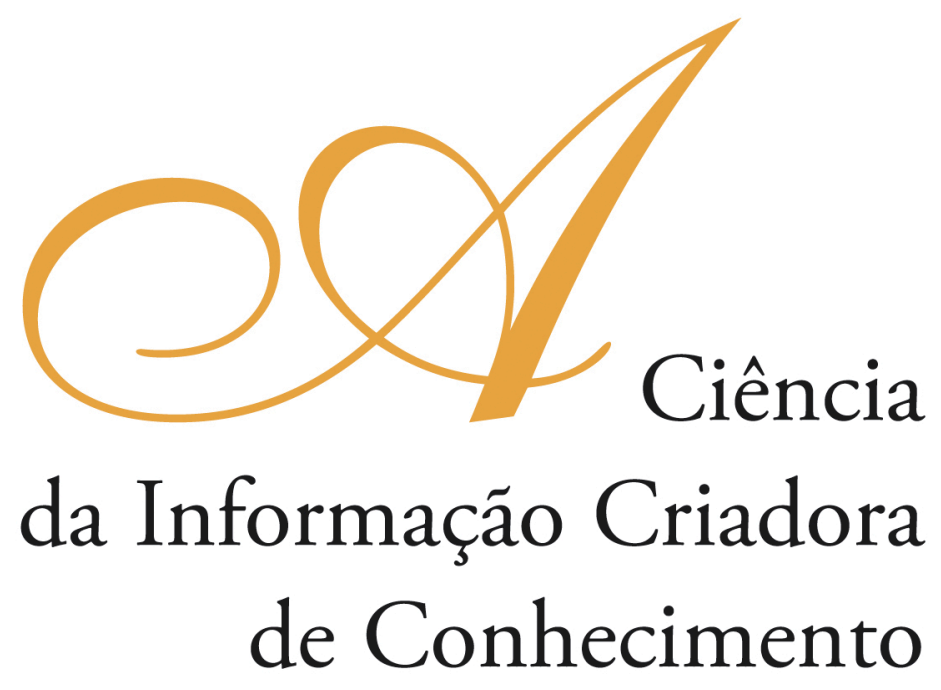

Vol. I

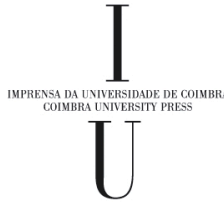

- COIMBRA 2009 


\title{
LA DIVULGACIÓN CIENTÍFICA EN EL ENTORNO UNIVERSITARIO
}

\author{
María Luisa Lascurain Sánchez \\ Universidad Carlos III de Madrid (Espanha) \\ Elías Sanz Casado \\ Universidad Carlos III de Madrid (Espanha)
}

\section{Resumen}

La divulgación científica tiene como objetivo fundamental acercar la ciencia a la sociedad, con el fin de incrementar su cultura científica e implicar a los ciudadanos en las actividades relacionadas con la investigación. En este proceso de socialización y comunicación del conocimiento juegan un papel fundamental las universidades, ya que esta tarea debería ser parte de su compromiso con la sociedad.

El objetivo de este trabajo es plantear la necesidad de incluir en los curricula de educación superior una materia transversal que facilite a los futuros investigadores los conocimientos básicos y las herramientas sobre la divulgación científica. Hoy en día son muy pocas las universidades que han introducido este tipo de cursos en su currículo.

\begin{abstract}
The main goal of popular science is to approach the science to the society, in order to increase its scientific culture implicating to the citizens in these research activities. Universities play a fundamental role in this socialization and communication process of knowledge, because this task should be part of its compromise with the society.

The objective of this work is the need to include a transverse course in the high education curricula providing to future scientists the basic knowledge and skills about popular science. Nowadays there are very few universities with this kind of courses in its curriculum.
\end{abstract}

La declaración de Budapest sobre la Ciencia y uso del saber científico (UNESCOICSU, 1999) apuesta por un debate "democrático, vigoroso y bien fundado sobre la producción y la aplicación del saber científico". Para llegar a ese debate es fundamental compartir la ciencia con los ciudadanos y fortalecer su confianza en la misma, que conozcan y comprendan la importancia que tienen la ciencia y la tecnología para la vida diaria, como instrumentos que han permitido el avance de la humanidad y por su capacidad de transformar la sociedad.

En la sociedad del conocimiento las personas deben estar no solo informadas, sino también formadas para desarrollar su capacidad crítica y de elección y para poder opinar en cuestiones científicas que le afectan como seres individuales y sociales. Esta visión de la ciencia como producto cultural es recogida por Calvo (2000) cuando afirma que "Hoy creemos de manera casi unánime que la divulgación de la ciencia y la tecnología es necesaria para el desarrollo cultural de un pueblo y es importante que ciertos hallazgos, experimentos, investigaciones y preocupaciones científicas se presenten al público y 
se constituyan en parte fundamental de su cultura en una sociedad profundamente impregnada por la ciencia y la tecnología como es la sociedad contemporánea”.

En este sentido, la divulgación científica es un instrumento para hacer equitativo el acceso a la cultura y al conocimiento. Y debe cumplir el papel de presentar no solo los resultados de la investigación sino acercar los temas para que el ciudadano pueda entender lo que es importante y porque.

Además, la divulgación contribuye a la rendición de cuentas que la investigación debe a la sociedad que la sustenta y de la que forma parte y que quiere saber los frutos de los recursos que se dedican a la investigación. El conocimiento de la ciencia contribuye a incrementar parte de dichos recursos, los recursos humanos, al lograr despertar el interés a futuros investigadores.

No está siendo fácil que la demanda del mensaje científico por parte de los usuarios potenciales del mismo sea importante. Para conocer la percepción de los españoles sobre la ciencia y sus actitudes hacia ésta, la Fundación Española para la Ciencia y la Tecnología (FECYT) ha realizado diversos encuestas (FECYT, 2002; 2004, 2006, 2008). El interés por los temas científicos se situaba en 2002 en un nivel de 5,7 sobre 10 y el nivel de información alcanzaba un valor de 4,4. Para el año 2004 se utilizó una escala sobre 5, en la que el interés alcanzaba un 2,82 y el nivel de información un 2,48. En cuanto a las actitudes, los encuestados en 2002 consideraban la ciencia como interesante (73\%), algo solidario $(57 \%)$, ético $(55 \%)$ e integrador (48\%), si bien también es percibida como algo frío por el 48\% (http:// www.fecyt.es/fecyt/home.do).

Sin embargo, según los datos observados en la última encuesta (2008), en relación a los años anteriores se aprecia un aumento en el interés que los españoles manifiestan tener sobre la ciencia y la tecnología. Según el estudio de FECYT (http://www.fecyt. es), el porcentaje de personas que asegura que no le interesa la ciencia ha disminuido respecto a 2006 del $36,6 \%$ al $31,7 \%$. También se ha observado un aumento significativo del número de ciudadanos que piensan que los beneficios de la ciencia y la tecnología son mayores que sus perjuicios. En este sentido, se ha elevado casi 10 puntos (del $44,8 \%$ en 2006 al $53,4 \%$ en 2008 ).

También habría que señalar, y esto es un resultado enormemente positivo del estudio, que en 2008 los encuestados consideran que ha mejorado la educación científico-tecnológica que ha recibido, puesto que las personas que consideraban que su educación científica había sido baja o muy baja, descendía del 65,5\% en 2004 hasta el $45,8 \%$ en 2008 .

Otro indicador importante que se analizó en la encuesta fue conocer la percepción que la sociedad española tenía de nivel su investigación respecto a Europa. Los datos han mostrado como ha variado positivamente esta percepción, puesto que en 2004 el $55,1 \%$ de los encuestados pensaba que la posición de España estaba muy atrasada respecto a Europa, y en 2008 este porcentaje disminuye hasta el 46,2\%.

La fuente de información más citada es la televisión (82,3\%). Sin embargo, para, un elevado porcentaje de jóvenes entre 15 y 24 años $(61,6 \%)$ y de 25 a 34 años $(50,4 \%)$ es Internet la fuente de información con mayor interés.

Respecto a los temas en los que se deberían invertir una mayor cantidad de fondos, destacan las fuentes energéticas (un 5\% más que en 2006) y el medio ambiente (4\% más en 2008 que en 2006). Sin embargo, la gran mayoría (más del 79\%) cree, como 
en la encuesta de 2006, que las mayores inversiones en investigación deberían dirigirse hacia los temas de medicina y salud.

Por tanto, consideramos que a pesar de los cambios que se empiezan a notar en la percepción social, la divulgación no puede hacerse bajos demanda, sino que necesita de una actitud proactiva para despertar el interés social por la ciencia. Esta actitud choca con la poca valoración del científico sobre esta necesidad y la ventaja que supone el reconocimiento social de su labor. La divulgación debería llegar a un importante segmento de la población, no solo difundiéndola entre los que ya muestran interés, sino también ganando adeptos, ya que la demanda nacerá del conocimiento.

La asimilación por parte de la ciudadanía de los conocimientos científicos no tiene correspondencia con el crecimiento exponencial de estos, advirtiéndose un desfase entre los descubrimientos y su vertido a la cultura popular (COTEC, 2006). Manuel Calvo (2002), fundador de la Asociación Espańola de Periodismo Científico, señala que el mayor problema de la divulgación científica es su retraso en comparación con los avances científicos y tecnológicos, y con su influencia tanto en el individuo como en los grupos sociales. Por ello, la brecha del conocimiento que se está generando entre los individuos que lo generan y aquellos a los que finalmente van dirigidas las aplicaciones provenientes de esos conocimientos es cada vez mayor. Podemos decir que en la historia de la humanidad nunca se ha llegado a tener tantos conocimientos como en el momento actual, y sin embargo, también es en este momento cuando de dan los mayores porcentajes de población excluida de esta riqueza. Gran parte de las sociedades actuales tiene una aproximación más mágica que humana sobre los conocimientos científicos que aplica en su vida diaria.

\section{La divulgación científica en el marco normativo español.}

Pese a su indiscutible valor cultural, la divulgación científica está escasamente contemplada en la normativa nacional o autonómica que regula la investigación, la ciencia y la tecnología.

El VI Plan Nacional de Investigación Científica, Desarrollo e Innovación Tecnológica para el periodo 2008/2011, que se define como un instrumento para el fomento y la coordinación de la investigación, incluye entre sus principios la puesta al servicio de la ciudadanía la I+D+i. Las líneas instrumentales del Plan no contemplan la divulgación, que aparece como transversal dentro de las acciones estratégicas. Estas acciones, cinco en total, se articulan mediante actuaciones específicas para cada una de ellas de cara a la consecución de una serie de objetivos entre los que figura el fomento de la cultura científica y tecnológica de la sociedad (Objetivo 6). Cómo objetivos específicos, el VI Plan incluye "aprovechar los nuevos formatos de comunicación para trasladar correctamente a la sociedad los avances científico-tecnológicos" (O6.1.), el desarrollo de estructuras que permitan la generación y promoción de cultura científica para "incrementar la cualificación de la opinión pública" (O6.2.) e "instalar nodos en red de comunicación científica en los agentes generadores de nuevos conocimientos para favorecer la comunicación social de la ciencia” (O6.3.) (Comisión Interministerial de Ciencia y Tecnología, 2007). 
La Estrategia Nacional de Ciencia y Tecnología con horizonte 2015 (ENCYT 2015), acordada en 2007, se entiende como un elemento de consenso y vertebración de las políticas de $\mathrm{I}+\mathrm{D}+\mathrm{i}$. Entre sus objetivos se plantea poner ésta "al servicio de la ciudadanía, del bienestar social y de un desarrollo sostenible, con plena e igual incorporación de la mujer". Incluye entre sus actuaciones la disposición de las condiciones adecuadas y necesarias para la difusión de la ciencia y la tecnología, con estructuras generadoras de información y promotoras de la formación intelectual; a través de certámenes, foros y premios; con recursos para estimular las oportunidades emprendedoras y con nuevos formatos de comunicación (Comisión Interministerial de Ciencia y Tecnología, 2007b).

Por su parte, el borrador del Anteproyecto de la nueva Ley de la Ciencia y la Tecnología (Ministerio de Ciencia e Innovación, 2009) únicamente trata aspectos relacionados con la regulación de la difusión de resultados y la cultura científica en tres artículos del Título III dedicado a los elementos para el impulso de la investigación científica y técnica y la transferencia del conocimiento y la difusión. El grupo de trabajo designado para la elaboración de esta norma, considera como novedad destacable el artículo sobre la publicación en acceso abierto que dispone que todos los investigadores cuya actividad científica este financiada con los presupuestos generales del Estado, estarán obligados a publicar en acceso abierto una versión electrónica en un plazo máximo de seis meses. Se insta a la Administración a que valore en sus procesos de evaluación las versiones electrónicas. El siguiente artículo se refiere a la mejora del acceso de la sociedad a la cultura científica y tecnológica mediante la realización distintas actividades (no las especifica). Por último, se dispone la creación por el Gobierno de la fundación del sector público estatal Museo Nacional de Ciencia y Tecnología.

A nivel autonómico, el IV Plan Regional de Ciencia y Tecnología de la Comunidad de Madrid (PRYCIT), incluye entre sus programas uno dedicado a la Ciencia y la Sociedad. Este programa recoge entre sus objetivos la promoción de la educación y la cultura científica en Europa, con la consiguiente sensibilización y diálogo con los ciudadanos y la elaboración de políticas científicas cercanas a estos. Como instrumento de coordinación del programa Ciencia y Sociedad del IV PRICYT está la Oficina de Información Científica, cuyo principal objetivo es favorecer el debate público sobre ciencia y tecnología (Dirección General de Universidades. Comunidad de Madrid, 2005).

\section{La divulgación en la universidad.}

La comunicación de la Comisión de las Comunidades Europeas sobre el papel de las universidades en la Europa del Conocimiento (COM (2003) 58/F, 2/5/2003) subraya la importancia de estas instituciones en los procesos fundamentales para el crecimiento de la Sociedad del Conocimiento, como son la producción de nuevos conocimientos, su transmisión a través de la educación y la formación, su divulgación a través de las tecnologías de la información y la comunicación y su empleo por medio de nuevos procedimientos industriales o de servicios. El comunicado incide en la idoneidad de las universidades para llevar a cabo esta labor a través de la investigación y la transferencia 
de sus resultados al sector industrial, de la educación y formación de profesionales y de investigadores y de su contribución al desarrollo regional y local.

Acercar la ciencia a la sociedad es, en parte, responsabilidad de los científicos, y muy especialmente de aquellos que reciben fondos públicos para su investigación, como es el caso de los que trabajan en universidades públicas, el Consejo Superior de Investigaciones Científicas, y otros organismos públicos de investigación, que deberían dedicar parte de su actividad a hacer accesible estos conocimientos que han generado a los ciudadanos.

El papel de las universidades en la divulgación científica es fundamental, puesto que una de las misiones de la Universidad, además de la docencia y la investigación es la transferencia del conocimiento que general a la sociedad. Actualmente, parte de esta misión es llevada a cabo a partir de la transferencia de tecnología que, mediante acuerdos, convenios, contratos, etc., realiza la Universidad con el mundo empresarial. Esta relación, que es fundamental para mantener un tejido industrial competitivo, aún no se ha influido en la relación con otros sectores sociales que necesitan recibir el conocimiento generado en los laboratorios universitarios.

La Universidad, como integrantes del sistema público de $\mathrm{I}+\mathrm{D}$, es responsable de la gran parte del conocimiento que se genera en la práctica totalidad de los países. En España, es responsable de algo más del $65 \%$ de los conocimientos. Por tanto, debería de favorecerse un compromiso social para que los científicos faciliten, transfieran y divulguen sus conocimientos en los distintos entornos sociales. Esto, será enormemente beneficioso para la sociedad en su conjunto, ya que ampliar el conocimiento científico que tiene el ciudadano, le hará más responsable de su actos, ayudándole a entender la delgada barrera existente entre obligaciones y derechos; y en definitiva a comprender, que en un mundo como el actual, con recursos limitados y serios problemas ecológicos y medioambientales, la ciencia puede ser una solución, siempre que sea entendida y asumida por la sociedad en su conjunto.

La universidad debe promover mecanismos y acciones que faciliten el acceso de la opinión pública a la investigación y a la docencia que realizan. De hecho ya lo están haciendo, puesto que la mayoría de universidades espańolas han creado oficinas de información científica, cuyo objetivo es facilitar los conocimientos que generan a la sociedad. Otra acción que debería realizar las universidades es la formación de universitarios con este tipo de conocimientos y habilidades, mediante cursos y programas específicos, con el fin de impregnar a los futuros profesionales e investigadores en la necesidad de introducir en la sociedad el interés por los tema científicos.

Por tanto, existiría un doble compromiso de la universidad en relación con la divulgación: Difundir entre la sociedad los resultados de su investigación y formar a los alumnos como futuros divulgadores de la investigación.

El primer aspecto corre a cargo de las Oficinas de Información Científica que cumplen la tarea de comunicación institucional y de transmisión de conocimientos científicos al gran público y que, como ya se ha comentado, con las que cuentan hoy en día la mayor parte de las universidades espańolas. Esta oficinas están formadas por personal cualificado en conocimiento y técnicas de comunicación social. En el caso concreto de la Universidad Carlos III de Madrid, la Oficina de Información Científica (http://www. uc3m.es/infocientifica), ha siso promovida por el Vicerrectorado de Investigación e integrada en el de Comunicación. Está oficina está integrada en la red de unidades de 
información científica de la Comunidad de Madrid y en la Red de Unidades de Cultura Científica y de Innovación (UCC+i) de la Fundación Espańola para la Ciencia y la Tecnología (FECYT) del Ministerio de Ciencia e innovación. Su objetivo fundamental es la difusión y divulgación a la sociedad de la $\mathrm{I}+\mathrm{D}+\mathrm{i}$ que se realiza en la UC3M. Además la OIC promueve, participa y organiza eventos y actividades divulgativos (por ejemplo, la Feria Madrid es Ciencia, la Semana de la Ciencia, y otros tipos de eventos divulgativos). Asimismo, esta oficina asesora a los profesores de la institución sobre como divulgar los resultados de sus investigaciones. También se encarga de redactar notas de prensa de aquellas investigaciones con mayor interés social, etc.

En cuanto al compromiso de formación de estudiantes e investigadores con los conocimientos y técnicas de la divulgación científica, la Universidad necesita realizar una labor intensa y sistemática con el fin de introducir a los estudiantes en este nuevo compromiso, así como favorecer un cambio gradual de las actitudes y aptitudes de los investigadores para afrontar este nuevo reto.

La difusión de su actividad mediante la publicación en revistas científicas especializadas es una práctica habitual entre los investigadores, ya que para ser valorados y creídos por la comunidad científica deben compartir sus resultados. Esto es lo que Ziman (1972) denominó el efecto colectivo o social de la ciencia. Sin embargo, otra cuestión es la divulgación, que supone crear un producto que llegue al gran público no especializado sin perder el necesario rigor científico. Algunos académicos consideran que se trata de una tarea menor que busca un tipo de protagonismo un tanto frívolo y consideran que el uso de una terminología asequible puede distorsionar el concepto mismo de la ciencia. A esto se une la escasa valoración que este tipo de actividades tiene en las distintas evaluaciones académicas o de investigación a las que se ven sometidos a las que se ven sometidos los investigadores, así como la falta de compensación económica. Todos estos factores junto con la escasa valoración, el factor tiempo y la falta de interés influyen en la poca participación en actividades de divulgación científica, como la Feria de Madrid es Ciencia o la Semana de la Ciencia, entre otros eventos (Rey Rocha, J. y Martín Sempere, M.J., 2007).

En relación con las aptitudes para divulgar, es preciso enseñarlas. No puede ser algo intuitivo ni voluntarista por parte de los investigadores, sino una labor institucionalizada y sistemática que requiere unos contenidos curriculares en la que se aúnen conocimientos procedentes de la realidad científica con los vinculados a las técnicas y metodologías implicadas en la comunicación como parte de la formación científica.

En muchos cursos de postgrado, en especial en los de máster de investigación y doctorado, se enseña a los alumnos a redactar artículos científicos entendiendo que debe formar parte del parte de su bagaje metodológico como futuros investigadores. Pero no se enseńa a transmitir esos conocimientos a la sociedad en general, es decir, a divulgar.

En una revisión de varios "libros blancos", hemos querido recoger como está contemplada la divulgación científica. En los libros blancos de los títulos de grado se recogen las propuestas de una red de universidades españolas, que si bien no son vinculantes, suponen un instrumento consensuado y de interés para la redacción de los diversos curricula. Son algunos los títulos que contemplan la divulgación entre las competencias que deben adquirir sus alumnos. Así en el título de Grado 
en Humanidades, se incluye la adquisición de métodos disciplinares en las técnicas de promoción, administración y divulgación de los bienes y actividades culturales. También en el Grado en Economía y Empresa se valoran las competencias relacionadas con la capacidad de divulgar las cuestiones económicas. La propuesta para Bioquímica y Biotecnología concreta más e incluye como competencia transversal la redacción de un artículo de divulgación que presente un contenido científico-técnico para su comprensión por lectores no expertos. Algunos títulos proponen entre sus objetivos la divulgación de la ciencia. Es el caso del grado en Geomática y Topografía, que en la visión global, multidisciplinar y multidimensional considera que el ingeniero no solo ha de conocer, sino también divulgar las tecnologías correspondientes a su campo. Otras titulaciones definen la divulgación dentro del perfil profesional propio de sus graduados. Es el caso del grado en Geografía y Ordenación del Territorio, de Estadística y Matemáticas, que además, en la distribución de contenidos propone que alguno de los cursos de libre configuración se pueda dedicar a la divulgación.

Aprender a divulgar supone aprender a traducir o interpretar e implica "recontexturalizar" para pasar de un conocimiento construido en contextos especializados a una situación comunicativa común para una audiencia lega y masiva, lo que requiere una reconstrucción del conocimiento para una audiencia diferente de la inicial (Blanco López, 2004). En palabras de Calvo (2002), los discursos de la divulgación se basan en la mediación ya que, si la comunicación entre el especialista y el público en general es imposible, hace falta un tercer elemento (el mediador) que traduzca el lenguaje del investigador para suscitar "interés, curiosidad y emoción”.

Por último, otra cuestión no resuelta en los debates sobre el tema es la de quién debe divulgar. ¿Deben hacerlo todos los investigadores o una parte de los mismos, por ejemplo, aquellos más vinculados con las tareas científicas? Y, ¿debe tratarse de periodistas científicos o de investigadores en materias concretas? Puede ser un científico con capacidad e interés por exponer unos resultados de investigación para un público no especializado, o un periodista con conocimientos científicos que pueda entender y transmitir al público una noticia científica.

En el programa que proponemos, la acción formativa se dirige a los futuros científicos que, desde cualquier área del conocimiento, desean aprender a divulgar los procesos y resultados de sus investigaciones, o las de otros colegas.

\section{Propuesta de programa.}

El objetivo del curso se dirige a la formación en la comunicación científica de alumnos universitarios de Grado. Para ello, se ha desarrollado un programa que se sustenta en cuatro ejes fundamentales: Emisor, Canal transmisor, Mensaje y Receptor

La planificación del curso, que se establece en 30 horas (3 créditos), se dividirá en una parte teórica, con la resolución de casos prácticos (20 horas) donde se dote al alumno tanto de los conocimientos básicos implicados en la actividad investigadora y su comunicación y divulgación, así como de herramientas necesarias para realizar esta actividad sin perder de vista la dimensión humanística. Finalmente los alumnos han de cursar una parte práctica (10 horas) en la que apliquen los conocimientos 
adquiridos. Esta parte práctica la realizan en la Oficina de Información Científica de la Universidad Carlos III de Madrid.

El programa consta de los siguientes módulos:

1.- La ciencia en la sociedad actual y su divulgación

En este módulo se aportará al alumno las nociones básicas del progreso científico, así como la importancia de las acciones de divulgación. El objetivo es que el alumno conozca los principales hitos científicos de nuestra historia que son referentes de cultura general, pero que configuran una base indispensable para la tarea del divulgador.

2.- El papel de los medios de comunicación en la divulgación científica

En este segundo módulo se muestran las herramientas, métodos, géneros y técnicas periodísticas aplicadas al periodismo científico. Se propone al alumno un análisis de esta especialidad en los medios de comunicación, con el fin de dotarle de instrumentos utilizados por los profesionales de la información a la hora de emprender su tarea divulgativa.

3.- Fuentes de Información de interés para la elaboración de los mensajes

Para divulgar es imprescindible acudir a las fuentes de información donde se registra el conocimiento científico. Por tanto es vital saber identificar, descifrar y usar correctamente estas fuentes de información. Los alumnos deben conocer por tanto las bases de datos científicas, las publicaciones científicas más importantes, los principales organismos públicos y privados de investigación, así como una relación de organismos dedicados a determinadas disciplinas.

4.- La divulgación científica realizada desde las universidades

La función del divulgador es trasladar a la sociedad el conocimiento y desarrollo tecnológico creado por la ciencia. El problema clásico de la divulgación es la complejidad del lenguaje científico, que debe ser "traducido" para el ciudadano. Para abordar esa tarea es básico conocer la terminología científica y realizar el esfuerzo de plasmarla en términos capaces de llegar a la sociedad, sin que por el camino se pierda el rigor del sentido original que le otorgó el científico.

En este módulo se mostrarán las oficinas de información científicas creadas por las universidades.

5.- La divulgación Científica ante un auditorio

El objetivo de este módulo es repasar los temas científicos más candentes de la actualidad, aquellos que tienen un mayor eco en los medios de comunicación y los que son de un mayor interés para la sociedad. Se trata de que el alumno tenga una perspectiva completa de la "novedad" sobre temas científicos y a la vez sepa dirigirse a un público presencial, con el fin de asegurar que llegue al mismo, de una manera atractiva y consistente su discurso divulgador.

\section{Referências bibliográficas}

Blanco López, A. (2004). Relaciones entre la educación científica y la divulgación de la ciencia. Revista Eureka sobre Enseńanza y Divulgación de las Ciencias, 1 (26), 70-86

Calvo, M. (2002). ¿Popularización de la ciencia o alfabetización cientifica? Ciencias, 66: 100105 
COM (2003) 58/F (5/2/2003). El papel de las universidades en la Europa del conocimiento. Bruselas: Comisión de las Comunidades Europeas.

COTEC (2006). Comunicar la Ciencia. Madrid: Fundación Cotec para la innovación tecnológica.

Echeverría, J. (2003). Percepción social de la ciencia y la tecnología en España -2002. Madrid: FECYT

Echeverría, J. (2005). Percepción social de la ciencia y la tecnología en España-2004. Madrid: FECYT

España. Fundación Española para la Ciencia y la Tecnología (FECYT). (2009). Consultado Ago 30, 2009, Disponible en http://www.fecyt.es/fecyt/home.do

España. Ministerio de Ciencia e Innovación (2009). Presentación del borrador de Anteproyecto de la nueva Ley de la Ciencia y la Tecnología, elaborado por el grupo de trabajo designado por la orden 1933/2008. Consultado Ago 30, 2009, Disponible en https://lcyt.fecyt.es/?page_id=354

España. Comisión Interministerial de Ciencia y Tecnología (2007). Plan nacional de Investigación Cientifica, Desarrollo e Innovación Tecnológica 2008-2011. Madrid: FECYT

España. Comisión Interministerial de Ciencia y Tecnología (2007). Estrategia nacional de ciencia y tecnología: ENCYT. Madrid: FECYT

Madrid (Comunidad Autónoma). Dirección General de Universidades e Investigación Madrid. Plan de Ciencia y Tecnología de la Comunidad de Madrid: un lugar para la ciencia y la tecnologia: IV PRICIT 2005-2008. Madrid: Comunidad de Madrid, Dirección General de Universidades

Rey Rocha, J. y Martín Sempere, M.J. (2007). Cicotec. El papel de los científicos en la comunicación de la ciencia y la tecnología a la sociedad: actitudes, aptitudes e implicación. Madrid: D.G. Universidades e Investigación, Consejo Superior de Investigaciones Científicas

UNESCO-ICSU (1999). Declaración de Budapest. Declaración sobre la Ciencia y el uso del saber cientifico. Consultado Jul 3, 2009, Disponible en http://www.oei.es/salactsi/budapestdec. htm

Ziman, J. M. (1972) El conocimiento público: Un ensayo sobre la dimensión social de la ciencia. México: Fondo de Cultura Económica. 